\title{
Validation of the OncoMasTR Risk Score in Estrogen Receptor-Positive/HER2-Negative Patients: A TransATAC study AC
}

Richard Buus ${ }^{1,2}$, Ivana Sestak ${ }^{3}$, Stephen Barron ${ }^{4}$, Tony Loughman ${ }^{4}$, Bozena Fender ${ }^{4}$, Cesar Lopez Ruiz ${ }^{4}$, Peter Dynoodt ${ }^{4}$, Chan-Ju Angel Wang ${ }^{4}$, Des O'Leary ${ }^{4}$, William M. Gallagher ${ }^{4,5}$, Mitch Dowsett ${ }^{1,2}$, and Jack Cuzick ${ }^{3}$

\section{ABSTRACT}

Purpose: To test the validity of OncoMasTR Molecular Score (OMm), OMclin1, and OncoMasTR Risk Score (OMclin2) prognostic scores for prediction of distant recurrence (DR) in estrogen receptor (ER)-positive/HER2-negative breast cancer treated with 5 years' endocrine therapy only and compare their performance with the Oncotype DX Recurrence Score (RS).

Experimental Design: OMm incorporates three master transcription regulator genes. OMclin1 combines OMm, tumor size, grade, and nodal status; OMclin2 incorporates OMm, tumor size, and nodal status. OMclin1 and OMclin2 were evaluated for 646 postmenopausal patients with ER-positive/HER2-negative primary breast cancer with $0-3$ involved lymph nodes in TransATAC. Patients were randomized to 5 years' anastrozole or tamoxifen without chemotherapy. RS was available in all cases. We used likelihood ratio- $\chi^{2}, \mathrm{C}$-index, and Kaplan-Meier analyses to assess prognostic information.

\section{Introduction}

Over $80 \%$ of primary breast cancers are estrogen receptor (ER)positive (1). After surgery, women with ER-positive disease typically receive 5 years of endocrine therapy, which markedly improves prognosis (2). A subset of patients, however, will remain at high risk of relapse if treated with endocrine therapy alone and identifying these is a major challenge in the management of breast cancer (3). Several prognostic gene signatures have been developed to assess residual risk after surgery and to guide treatment decisions including the 21-gene Oncotype DX Recurrence Score (RS), the intrinsic subtype-based Prosigna PAM50 Risk of Recurrence (ROR) Score, the Breast Cancer

${ }^{1}$ The Breast Cancer Now Toby Robins Research Centre at The Institute of Cancer
Research, London, United Kingdom. ${ }^{2}$ Ralph Lauren Centre for Breast Cancer
Research, Royal Marsden Hospital, London, United Kingdom. ${ }^{3}$ Centre for Cancer
Prevention, Wolfson Institute of Preventive Medicine, Queen Mary University of
London, London, United Kingdom. ${ }^{4}$ OncoMark Limited, Dublin, Ireland. ${ }^{5}$ UCD
School of Biomolecular and Biomedical Science, UCD Conway Institute, Univer-
sity College Dublin, Belfield, Dublin, Ireland.

Note: Supplementary data for this article are available at Clinical Cancer Research Online (http://clincancerres.aacrjournals.org/).

Corresponding Author: Richard Buus, The Institute of Cancer Research, London, SW3 6JB, United Kingdom. Phone: 4420-7808-2619; Fax: 4420-7808-2808; E-mail: richard.buus@icr.ac.uk

Clin Cancer Res 2019;XX:XX-XX

doi: 10.1158/1078-0432.CCR-19-0712

(C)2019 American Association for Cancer Research.
Results: OMm, OMclin1, and OMclin2 were highly prognostic for prediction of DR in years 0-10 among all patients [likelihood ratio (LR) $-\chi^{2}=25.4,48.7$, and 45.0 , respectively, all $P<0.001$; Cindex $=0.67,0.71$, and 0.71 , respectively], compared with RS (LR- $\chi^{2}$ $=18.8 ; P<0.001 ; \mathrm{C}$-index $=0.63)$. All three scores provided significant additional prognostic value beyond clinical treatment score, Nottingham Prognostic Index, and Ki67. OMclin1 and OMclin2 categorized 190 and 267 node-negative patients as low risk (DR rates: $2.9 \%$ and $4.9 \%$, respectively). In comparison, RS categorized 296 node-negative patients as low-risk and 128 patients as intermediate-risk (DR rate: $6.6 \%$ and $17.3 \%$, respectively).

Conclusions: OMm, OMclin1, and OMclin2 were highly prognostic for early and late DR in women with early-stage ER-positive breast cancer receiving 5 years' endocrine therapy. In TransATAC, OMclin1 and the OncoMasTR Risk Score (OMclin2) were superior to RS in identifying patients at increased risk of DR.

Index (BCI) combining the molecular grade index with a two-gene ratio, the 12-gene EndoPredict (EPclin), and the 70-gene MammaPrint score (4-8).

While all these signatures provide prognostic information on breast cancer recurrence, there is little overlap between the genes. This suggests that there may be upstream coregulation by other genes that are more fundamentally associated with breast cancer recurrence. The OncoMasTR prognostic gene signature was derived by identifying transcriptional components that regulate the genes contained within existing prognostic signatures. A novel bioinformatic approach (ARACNe: Algorithm for the Reconstruction of Accurate Cellular Networks) identified a shared network of 10 master transcriptional regulators (MTR) underpinning two existing prognostic gene signatures (9): the 231 genes from which the 70 -gene MammaPrint was derived (8) and the 207 genes from which the 97-gene Genomic Grade Index was derived (ref. 10; Supplementary Fig. S1). Chromatin immunoprecipitation studies showed that the MTRs bind, and directly regulate, the promoters of a set of proliferation-associated genes, many of which are highly enriched in breast cancer prognostic signatures. In addition, MTRs were found to be prognostic at both mRNA and protein levels (11).

The OncoMasTR Molecular Score (OMm) was identified as the most prognostic combination of these MTRs, FOXM1, PTTG1, and ZNF367, each of which has been demonstrated to play a critical role in cell proliferation and other key features of malignancy (12-15). OMclin 1 combines OMm with nodal status, tumor size, and grade. OMclin2 (final OncoMasTR Risk Score) is a simpler form of OMclin1 that excludes tumor grade. Both OMclin1 and OMclin2 stratify patients into low- and high-risk groups. 


\section{Translational Relevance}

In this study we report the validation of the OncoMasTR Risk Score for estrogen receptor-positive $\left(\mathrm{ER}^{+}\right) / \mathrm{HER} 2^{-}$primary breast cancer in 646 postmenopausal patients treated with 5 years' tamoxifen or anastrozole. The OncoMasTR Risk Score combines the expression of three master transcription regulators (MTR) with nodal status and tumor size. The MTRs (FOXM1, PTTG1, and ZNF367) regulate previously known sets of prognostic genes and have well-characterized functional roles in several aspects of cancer. The signature categorizes patients into the clinically actionable low- and high-risk groups. We found that the prognostic information from the OncoMasTR Risk Score was more accurate than that from the Oncotype DX Recurrence Score, the most widely used prognostic signature in $\mathrm{ER}^{+}$breast cancer.

The main objective of this study was to clinically validate the OMm, OMclin1, and OMclin2 prognostic scores in an independent dataset (TransATAC) and to compare their performance with that of the Oncotype DX RS. TransATAC, the translational substudy of the Arimidex, Tamoxifen, Alone or in Combination trial (ATAC; ref. 16) is a large collection of well-characterized samples from postmenopausal patients with ER-positive, HER2-negative primary breast cancer treated with 5 years' of endocrine therapy only. It served as a validation cohort for the Oncotype DX RS, Prosigna PAM50 ROR, BCI, and EPclin scores (17-20).

\section{Materials and Methods \\ Study population}

Samples were available from TransATAC (16) where RNA was extracted by Genomic Health Inc. (GHI; ref. 17). Eligibility for this study required hormone receptor-positive, HER2-negative disease, chemotherapy-naïve, RS available, and sufficient residual RNA for OncoMasTR analysis.

\section{Analytic methods}

There were sufficient quantities of residual RNA available from 702 patient samples. To establish whether RNA extracted by GHI was suitable to obtain reliable OMm scores a pilot study was conducted. From paired tissue sections of 108 patient samples, RNA was extracted using the process validated for the OMm assay and individual gene measurements and OMm scores were compared with that obtained from GHI-extracted RNA.

RNA (100-200 ng) was used to measure expression of the 6 genes (the three genes of interest and three reference genes; GAPDH, GUSB, and TFRC) constituting OMm by RT-qPCR performed by OncoMark. Data from 14 of the 702 samples did not meet the prespecified OncoMasTR data quality criteria and were excluded from statistical analyses. All genes were measured in triplicate. The relative expression level of each OMm gene of interest $(\Delta C \mathrm{q}$ GOI) was calculated as follows: $\triangle C \mathrm{q}$ GOI $=$ GeometricMean(Mean(GAPDH triplicates), Mean(GUSB triplicates), Mean(TFRC triplicates)) - Mean(GOI triplicates). The three $\Delta \mathrm{Cq}$ values were then used to calculate the continuous molecular risk score according to the OMm prognostic algorithm. Thresholds for the numeric score to stratify patients into low- and high-risk groups were based on sensitivity and specificity in the training cohort. For OMclin1, the threshold was the numeric score value that maximized the sum of sensitivity and specificity (Youden
Index). The resulting risk groups had Kaplan-Meier distant recurrence (DR) rates of $4 \%$ and $33 \%$ in the training cohort. For OMclin2, the threshold was the numeric score value at which both sensitivity and specificity were 0.7 . The resulting risk groups had Kaplan-Meier DR rates of $8 \%$ and $36 \%$ in the training cohort. In TransATAC, unscaled OMclin1 and OMclin2 scores ranged between -4.13 and 2.19 and -4.60 and 1.65 , respectively. To present the scores in a more intuitive, user-friendly way the scores were rescaled to range between 0 and 10 with the following equations: rescaled OMclin $1=$ raw score $\times 1.2+$ 6.0258; rescaled OMclin $2=$ raw score $\times 1.2+7.0059$. In each case, the scaling resulted in the high-low risk cutoff having a value of 5 . The linear transformations retained the shape of the distribution of the unscaled scores.

These analytic methods were performed by OncoMark blinded to clinico-pathologic information and clinical outcome.

\section{Study endpoints}

The prospectively defined primary endpoint was distant recurrence-free survival defined as the interval from diagnosis until DR, or death due to breast cancer. Contralateral breast cancer and death due to causes other than breast cancer were censoring events. Death due to breast cancer where a recurrence had not been recorded was treated as an event with the event date being the date of death.

\section{Statistical analyses}

Analyses were performed using 10-year median follow-up outcome data (16) according to a prespecified statistical analysis plan approved by the Long-term Anastrozole versus Tamoxifen Treatment Effects committee and OncoMark Ltd. before data analysis.

Our stepwise primary objectives were to assess whether OMm had statistically significant prognostic information for 10-year DR as a continuous variable and as a categorical variable. If so, we would test OMclin1 as continuous score and as categorical variable. Secondary analyses included testing the prognostic value of OMm and OMclin1 in early ( $0-5$ years) and late ( $5-10$ years) settings, in patients divided into subgroups by nodal status, and to test whether additional prognostic information was provided when added to the clinical treatment score (CTS), Nottingham Prognostic Index (NPI), and Ki67 measured by IHC. Subsequently, OMclin2 was added to the analysis plan due to further optimization of clinico-pathologic features and was subjected the same analyses as OMclin1.

Briefly, Cox proportional hazards regression models were fitted and hazard ratios (HR) and 95\% confidence intervals (CI) were estimated. Likelihood ratio (LR) tests were used for hypothesis testing. As previously reported, the CTS integrated the prognostic information from nodal status, tumor size, histopathologic grade, age, and type of endocrine treatment (21). All statistical tests were two-sided, a $P$ value of less than 0.05 was regarded as statistically significant. All statistical analyses were performed with STATA version 13.1 at the Queen Mary University of London (London, United Kingdom). This study was approved by the South-East London Research Ethics Committee, and all patients included gave informed consent. This study meets the Reporting Recommendations for Tumor Marker Prognostic Studies (REMARK).

\section{Results}

Sample availability is shown in the CONSORT diagram (Fig. 1). An OncoMasTR Molecular Score was obtained for 688 patients, of whom we are reporting results for node-negative and node 1-3 positive patients in this study (i.e., excluding those with four or more positive nodes). OMm data were available for 648 samples, and 646 of these had 
Figure 1.

CONSORT diagram of the availability of samples for analysis from the ATAC trial. PgR, progesterone receptor; LN, lymph node.

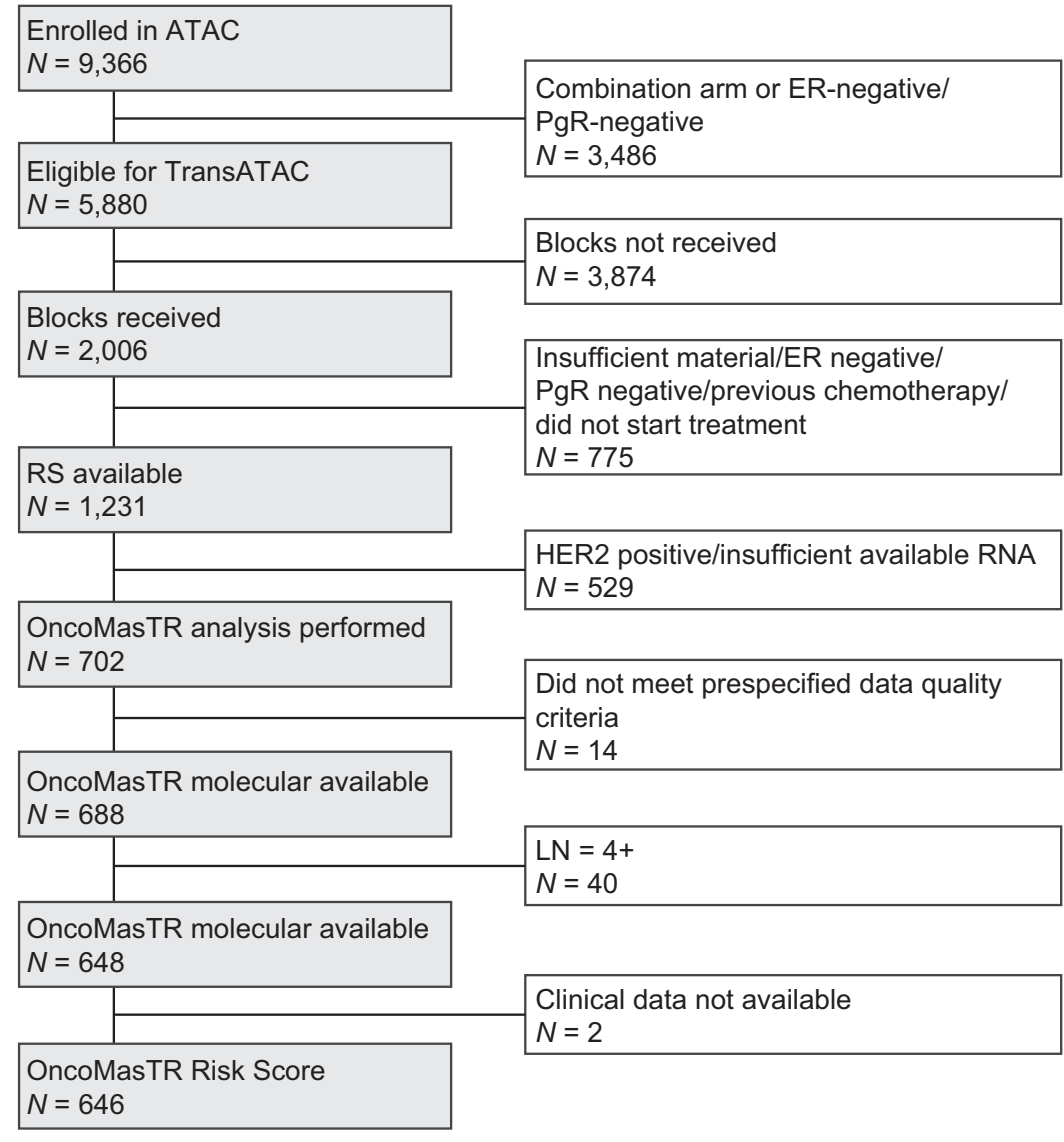

data on OMclin1 and OMclin2 (due to missing clinico-pathologic data). The characteristics of this TransATAC cohort are presented in Supplementary Table S1. A total of 88 DRs were recorded within the 10 -year median follow-up period. There were 50 DRs in node-negative women $(n=482)$ and 38 DRs were detected in women with nodepositive disease $(n=164)$.

The pilot study demonstrated the suitability of the preextracted RNA for OMm analysis (Supplementary Fig. S2). Pearson correlation coefficients for FOXM1, PTTG1, and ZNF367 were 0.93, 0.81, and 0.80 , respectively; for $\mathrm{OMm}$ Pearson correlation coefficient was 0.91 .

\section{Univariate analyses of continuous prognostic scores}

OMm, OMclin1, and OMclin2 were highly prognostic for the whole population across 10 years, with OMclin1 and OMclin2 providing substantially more information than the molecular OMm score alone $\left(\right.$ LR- $\chi^{2}:$ OMm $=25.4 ;$ OMclin $1=48.7$, OMclin2 = 45.0; Table 1). $\mathrm{OMm}$ was also significantly prognostic in the early and late settings and in node-negative patients, however, OMm provided no significant information in the node-positive population. OMclin1 and OMclin2 were significantly prognostic across all subpopulations examined, except for $0-5$ years in the node-positive subgroup, which was not significant. OMm, OMclin1, and OMclin2 also provided significantly more prognostic information in $0-10$ years than RS in all patients (LR$\chi^{2}: \mathrm{RS}=18.8$ ). This was driven by the node-negative group where RS was also inferior. However, in node-positive patients, OMm and RS were equally uninformative. OMclin 1 and OMclin 2 were also highly prognostic for the prediction of late DR $\left(\mathrm{LR}-\chi^{2}=25.6\right.$ and LR- $\chi^{2}=$ 25.1, respectively, $P<0.001)$.
C-index statistics calculated for the scores showed superior model fit of OMm, OMclin1, and OMclin2 when compared with RS (C-index: $\mathrm{OMm}=0.666$; OMclin1 $=0.708 ;$ OMclin2 $=0.713 ; \mathrm{RS}=0.634$ ).

\section{Multivariate analyses of continuous prognostic scores}

Multivariate comparisons with CTS are shown in Table 1. Across 10 years in the overall population OMm, OMclin1, OMclin2, and RS all provided significantly more prognostic information beyond that of the CTS, with RS providing the least amount of information (LR- $\Delta \chi^{2}$ : 13.9; 15.8; 15.8; and 10.7 for OMm, OMclin1, OMclin2, and RS, respectively). Similar results were observed in the node-negative subgroup. However, in node-positive patients none of the scores added significant prognostic value to CTS. OMm, OMclin1, and OMclin2 also added significant information to CTS in the early and late settings in the overall population. This was led by their good performance in the node-negative cohort, in contrast to the nodepositive group where none of the signatures remained significant when added to CTS. Consistent with the analysis of the continuous scores, Kaplan-Meier analysis of CTS (categorized at the median) versus a CTS+OMclin2 composite score (categorized at the median) showed that CTS+OMclin2 provided better separation than CTS alone in node-negative patients but not in the node-positive group (Supplementary Fig. S3).

A similar pattern emerged in the multivariate comparisons with NPI: OMm, OMclin1, and OMclin2 all added significant prognostic information to NPI in all patients across 10 years (LR- $\Delta \chi^{2}: 9.4 ; 11.5$; and 13.7 for OMm, OMclin1, and OMclin2, respectively; Supplementary Table S2). Similar to the comparisons with CTS, no significant added information to NPI was found in the node-positive subgroup 


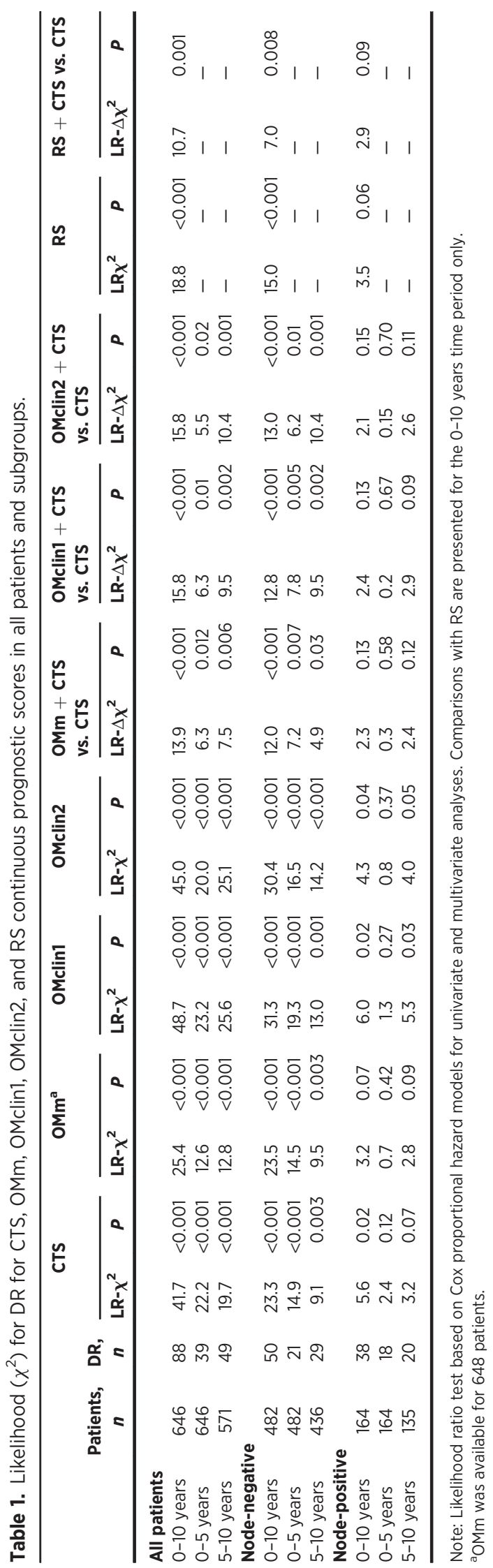

analyses. OMm, OMclin1, and OMclin2 also added significant prognostic information to Ki67 in all patients (LR- $\Delta \chi^{2}: 9.4 ; 30.5$; and 27.1 for OMm, OMclin1, and OMclin2, respectively; Supplementary Table S3). No significant added information was found for OMm in the early setting (LR- $\Delta \chi^{2}: 2.5$ for OMm) contrary to after 5 years where it provided additional information (LR- $\Delta \chi^{2}: 7.2$ for OMm).

\section{Categorical analyses}

Using predefined cutoffs, the distribution between low- and highrisk groups for OMclin1 was 219 (33.9\%) versus 427 (66.1\%) patients and for OMclin2 it was $305(47.2 \%)$ versus 341 (52.8\%) patients (Table 2, Fig. 2). The mean DR rates at 10 years were $4.0 \%(2.0-$ 7.9) versus $21.2 \%$ (17.3-25.7) for OMclin 1 and $5.4 \%$ (3.3-8.8) versus $24.3 \%$ (19.8-29.7) for OMclin2 in the low- and high-risk groups, respectively. Greater HR was found between the high- and low-risk groups for OMclin1 than for OMclin2: 5.8 (2.8-12.0) versus 4.9 (2.88.6) but the difference was not statistically significant. More patients were categorized as low risk by RS $(389,60.2 \%)$ than by OMclin 1 and OMclin2, however at $9.9 \%$ the RS low-risk group had substantially greater DR risk than the low-risk groups by OMclin1 (4.0\%) and OMclin2 (5.4\%; Table 2). We combined the RS intermediateand high-risk groups to create an RS non-low-risk group. OMclin1 and OMclin2 categorized 427 and 341 patients into high-risk category compared with 257 in the non-low group of RS. The corresponding DR rates for the three groups were similar at $21.2 \%$, $24.3 \%$, and $23.4 \%$, respectively. Figure 3 shows the continuous relationship between OMclin1, OMclin2 scores, and 10-year DR risk. OMclin2 corresponds to higher risk than OMclin1 at the cutoff point for risk categorization.

In women with node-negative disease, OMclin1 identified $39.4 \%$ of women as low risk with a 10 -year DR risk of $2.9 \%(1.2-6.8)$, which was significantly lower compared with those categorized as high risk [10year DR risk: $17.3 \%$ (13.2-22.6); $\mathrm{HR}$ of high risk versus low risk HR = $6.5(2.6-16.3)]$. OMclin2 categorized $55.4 \%$ of patients as low risk with a 10 -year DR rate of $4.9 \%(2.8-8.5)$ compared with $19.9 \%(14.8-26.4)$ in the high-risk group [HR of high risk versus low risk $=4.3(2.3-8.3)$ ]. This compared with 296 (61.4\%) low-risk patients by RS with a 10-year DR rate of 6.6\%. In addition, we applied the cutoff points for RS used in the TAILORx trial (Tx) to assign patients to treatment (22). In the node-negative group 145, 240, and 97 patients were categorized into the Tx low ( $\mathrm{RS}<11$ ), Tx intermediate (RS 11-25), and Tx high (RS $>25$ ) groups, respectively, with DR rates of $9.3 \%, 8.2 \%$, and $23.5 \%$, respectively.

In node-positive disease, the HR for OMclin1 low versus high risk was nonsignificant at 2.9 (0.9-9.5); however, for OMclin2, the HR was significant at $4.2(1.3-13.6)$.

Patient scores by RS plotted against OMm, OMclin1, and OMclin2 scores is presented in Fig. 4. Score distribution by nodal status was different for OMclin1 and OMclin2 with a shift of node-positive patients toward higher risk, not seen for RS and OMm. Spearman rho correlation coefficient was similarly modest across the scores: RS versus $\operatorname{OMm}(\rho=0.30)$, RS versus OMclin1 $(\rho=0.34)$, and RS versus OMclin2 $(\rho=0.29)$. Similar correlation coefficients were found in the node-negative subgroups for the three comparisons: RS versus OMm $(\rho=0.28)$, RS versus OMclin1 $(\rho=0.34)$, and RS versus OMclin2 $(\rho=0.29)$.

\section{Discussion}

The currently available commercial prognostic signatures for ER-positive breast cancer were trained and discovered using gene 


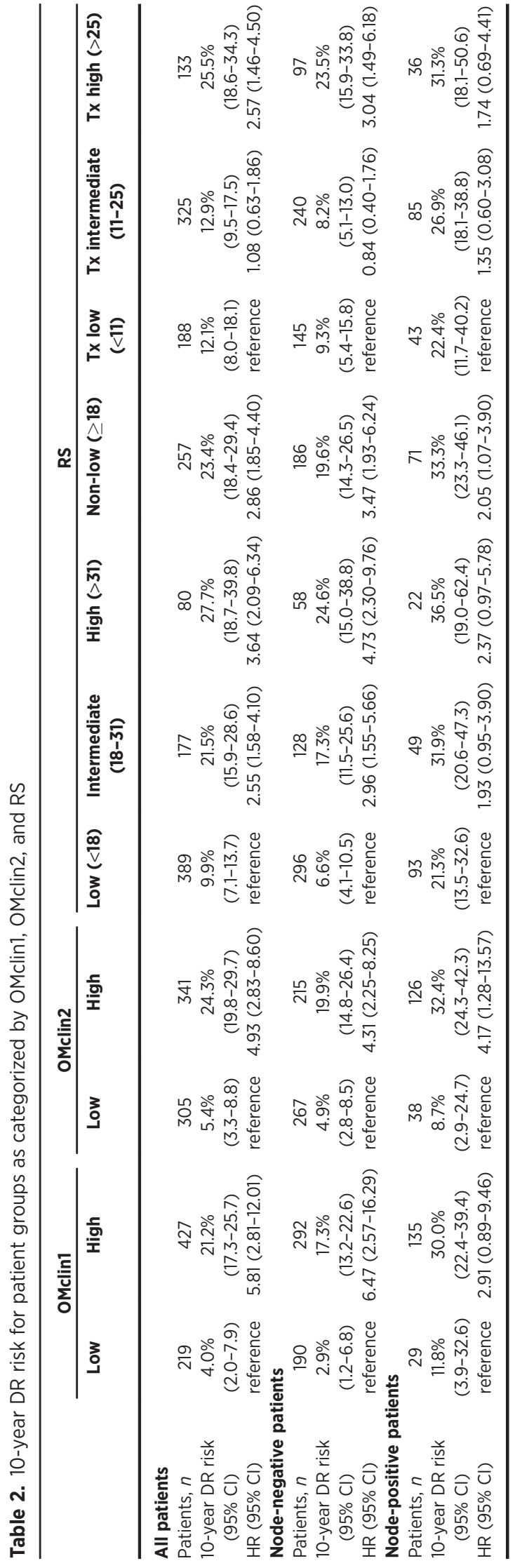

expression profiling of breast cancer samples and have generally resulted in panels including a large number of genes. OMm was discovered through querying the dependencies between genes from two well-validated breast cancer prognostic signatures, which resulted in the identification of a shared transcriptional network of MTRs upstream of the signatures $(9,11)$. FOXM1, PTTG1, and ZNF367 have been demonstrated to play critical roles in tumor progression. The FOXM1 (Forkhead Box M1) gene encodes a forkhead transcription factor, which controls cell proliferation, maintenance of stem cell properties, invasion and metastasis, and is associated with poor prognosis in ER-positive patients treated with tamoxifen (12). PTTG1 (pituitary tumor transforming gene 1) promotes tumor metastasis through enhancing the proliferation, invasion, and metastasis of cancer cells (13). Elevated levels of its protein product, securin, is an independent prognosticator of breast cancer-specific survival even among invasive ductal breast carcinoma with low Ki-67 positivity (14). ZNF367 (zinc finger protein 367, also known as ZFF29 and CDC14B) is found to be overexpressed in a variety of endocrine cancers. It is reported to inhibit in vitro and in vivo growth, cellular invasion, migration, and adhesion to extracellular proteins, suggesting a protective role by inhibiting cancer progression (15). Thus, biologically, the signature consists of genes that regulate previously known prognostic genes and have identified functional roles in several hallmarks of cancer including cell proliferation, invasion, and metastasis. The clinically applicable signature incorporates clinico-pathologic information, and categorizes patients into clinically actionable low- or highrisk groups.

In this TransATAC study, we showed that the OMm, OMclin1, and the OMclin2 have statistically significant prognostic ability for DR in breast cancer patients with ER-positive, HER2-negative disease who received 5 years' of endocrine therapy. All three scores were significantly prognostic as continuous variables in the early and late settings and in the node-negative groups. However, no substantial prognostic information was found in the node-positive group. This might be at least in part due to the exclusion of patients with 4 or more involved nodes in this validation study and the associated lower number of events in this group. OMclin1 and OMclin2 provided a similar degree of prognostic information and both outperformed the purely molecular OMm score. This finding underlines the prognostic value of clinico-pathologic features and the importance of predictors incorporating them for accurate prognostics. The exclusion of grade for OMclin2 did not substantially affect its performance. Comparing the molecular-only scores in the 10 -year follow-up period, OMm was found to be moderately superior to RS suggesting that the 3 MTR genes might be better at capturing key aspects of breast cancer recurrence than the RS algorithm made up of 16 prognostic genes. However, the limited size of the study population and this modest difference means that actual superiority of the OMm should be regarded as uncertain.

To perform a fair comparison of the molecular RS score with OMclin1 and OMclin2, we examined the added prognostic information of these scores to CTS. Both OMclin1 and OMclin2 were found to be modestly superior to RS in the overall and in the node-negative groups in this population; however, none of the three signatures added value to CTS in the node-positive group.

Risk categorization by OMclin1 and OMclin2 on the basis of predefined cutoffs showed a clear separation of low- and high-risk groups in the overall and node-negative groups. In node-positive patients, OMclin1 showed reduced prognostic performance; however, OMclin2 remained significantly prognostic. Previous data have shown the reduced prognostic power of RS in the late period was partly due to high ER expression being associated with poor prognosis after 
Buus et al.
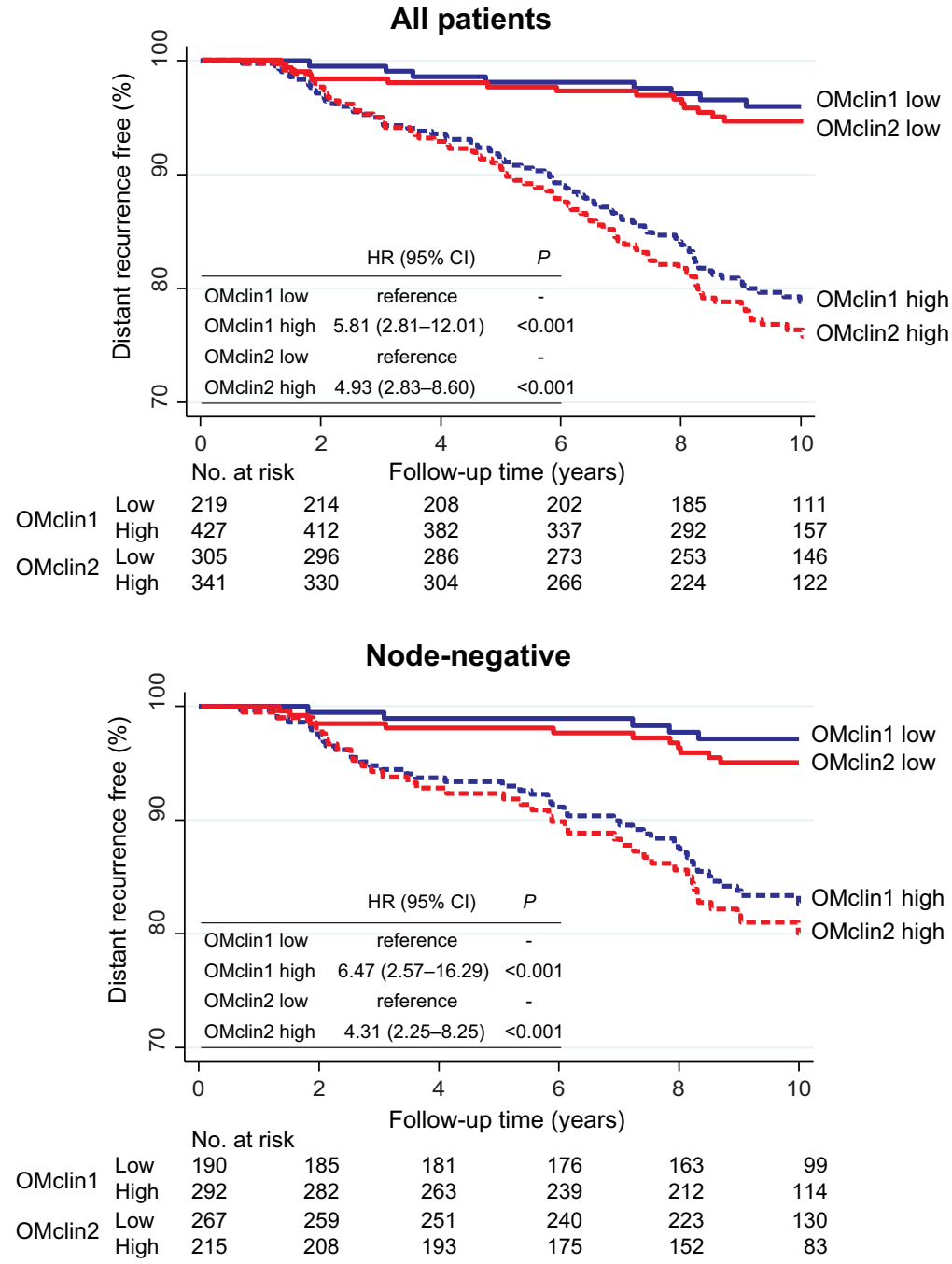

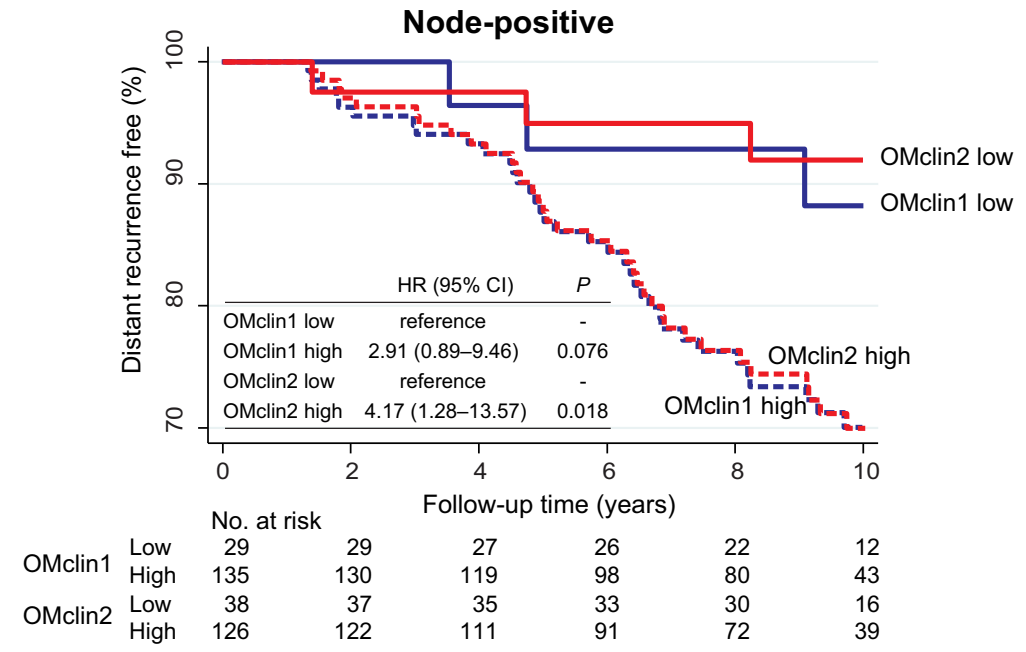

\begin{tabular}{|c|c|c|c|c|c|c|c|}
\hline \multirow{3}{*}{ OMclin1 } & & \multicolumn{2}{|c|}{ No. at risk } & 年 & 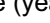 & & \\
\hline & Low & 190 & 185 & 181 & 176 & 163 & 99 \\
\hline & High & 292 & 282 & 263 & 239 & 212 & 114 \\
\hline OMclin? & Low & 267 & 259 & 251 & 240 & 223 & 130 \\
\hline & High & 215 & 208 & 193 & 175 & 152 & 83 \\
\hline
\end{tabular}

Figure 2.

Kaplan-Meier plots for 10-year DR for OMclin1 and OMclin2 risk groups in all patients, node-negative patients, and nodepositive patients. The numbers of patients at risk in each group at various time points are given below each graph. 
Figure 3.

Probability of DR as a continuous function of OMclin1 and OMclin2 and $95 \% \mathrm{Cl}$ (dashed lines). Vertical line represents cutoff point of 5 for low and high risk.

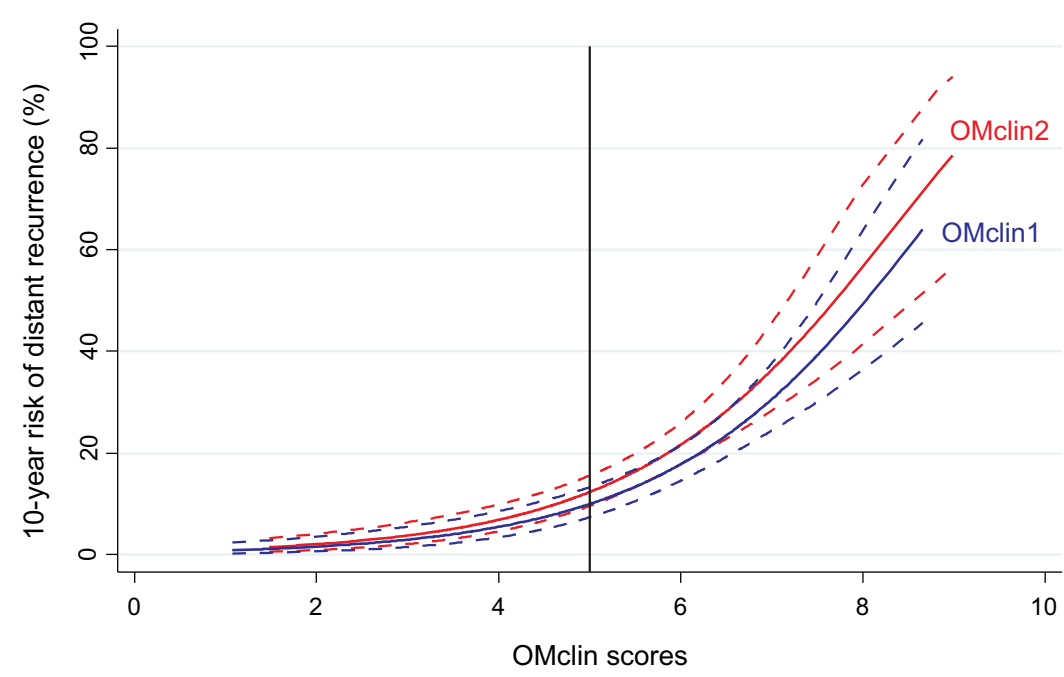

resent ATAC per se. The prognostic performance of RS in this study (both univariate and multivariate analyses with CTS) was lower than that reported previously in the more complete TransATAC cohort (17). Supplementary Table S4 shows the demographic differences between those included in this study and those that were not included from the earlier study. Of particular note was the difference in performance noted for RS in the two studies. This may be partly explained by our exclusion of HER2-positive cases from this study because contemporary use of molecular signatures is confined to HER2-negative disease. Also, the node-positive group in this analysis was restricted to those with 1-3 positive nodes. In addition, because of these eligibility criteria and reduced sample availability, fewer samples were analyzable in this study compared with the previously published TransATAC studies. This inevitably leads to reduced $\chi^{2}$ values.

On the basis of these findings, further validation studies are warranted to assess some key questions, such as (i) Is the performance of OncoMasTR compared with RS found here confirmed in other cohorts? (ii) With sufficient sample size, does OncoMasTR add significant prognostic value to clinical information among lymph node positive patients? (iii) Is OncoMasTR predictive for therapy benefit? (iv) Is OncoMasTR prognostic and/or predictive among premenopausal women?
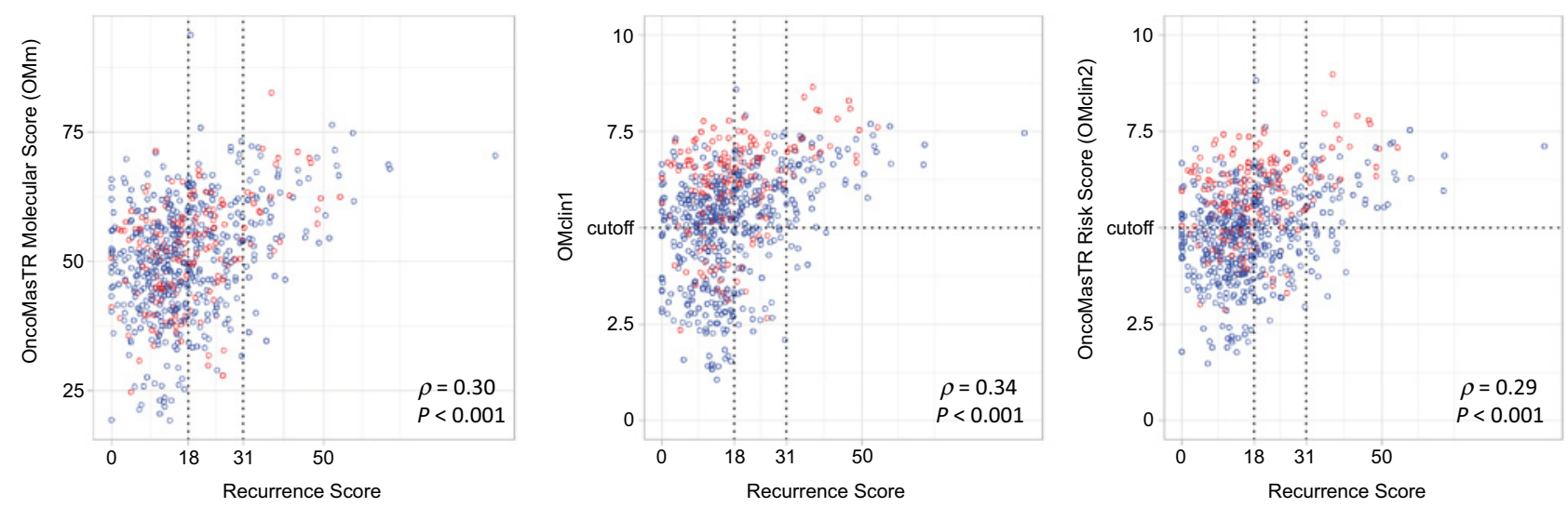

\section{Figure 4.}

Distribution of prognostic scores. Scatterplot of RS with OMm, OMclin1, and OMclin2 for 646 patients. Blue circles, node-negative patients; red circles, node-positive patients; dashed line, cutoff points for risk stratification. Spearman rho and $P$ values are presented. 
In summary, our study confirmed the independent prognostic ability of OMm, OMclin1, and OMclin2 in postmenopausal patients with ER-positive breast cancer given 5 years' of endocrine therapy. Furthermore, we showed that on the basis of a modest enhancement of $\mathrm{OMm}$ over RS and also on the incorporation of clinical factors OMclin1 and the simpler OncoMasTR Risk Score (OMclin2) were superior in this population to Oncotype DX RS in identifying patients at increased risk of DR. Further study is required to confirm these findings in other cohorts.

\section{Disclosure of Potential Conflicts of Interest}

I. Sestak reports receiving other remuneration from Myriad Genetics, NanoString Technologies, and Pfizer Oncology. S. Barron is an employee/paid consultant for OncoMark Ltd. T. Loughman is an employee/paid consultant for OncoMark Ltd. B. Fender is an employee/paid consultant for OncoMark Ltd. C.L. Ruiz is an employee/paid consultant for OncoMark Ltd. P. Dynoodt is an employee/paid consultant for OncoMark Ltd. C.-J.A. Wang is an employee/paid consultant for OncoMark Ltd. D. O'Leary is an employee/paid consultant for and holds ownership interest (including patents) in OncoMark Ltd. W.M. Gallagher is an employee/paid consultant for and holds ownership interest (including patents) in OncoMark Ltd. M. Dowsett reports receiving speakers bureau honoraria from Myriad and NanoString, and reports receiving other remuneration from the Institute of Cancer Research Rewards for Inventors. J. Cuzick reports receiving commercial research grants from AstraZeneca, is an unpaid consultant/advisory board member for Merck, and reports receiving other remuneration from Cancer Research UK. No potential conflicts of interest were disclosed by the other authors.

\section{Authors' Contributions}

Conception and design: R. Buus, I. Sestak, C.-J.A. Wang, D. O'Leary, W.M. Gallagher, M. Dowsett, J. Cuzick

\section{References}

1. Dodson A, Parry S, Ibrahim M, Bartlett JMS, Pinder S, Dowsett M, et al. Breast cancer biomarkers in clinical testing: analysis of a UK NEQAS ICC \& ISH database containing results from 199,300 patients. J Pathol Clin Res 2018;4: 262-73.

2. Dowsett M, Forbes JF, Bradley R, Ingle J, Aihara T, Bliss J, et al. Aromatase inhibitors versus tamoxifen in early breast cancer: patient-level meta-analysis of the randomised trials. Lancet 2015;386:1341-52.

3. Pan H, Gray R, Braybrooke J, Davies C, Taylor C, McGale P, et al. 20-year risks of breast-cancer recurrence after stopping endocrine therapy at 5 years. N Engl J Med 2017;377:1836-46

4. Paik S, Shak S, Tang G, Kim C, Baker J, Cronin M, et al. A multigene assay to predict recurrence of tamoxifen-treated, node-negative breast cancer. $\mathrm{N}$ Engl J Med 2004;351:2817-26

5. Nielsen TO, Parker JS, Leung S, Voduc D, Ebbert M, Vickery T, et al. A comparison of PAM50 intrinsic subtyping with immunohistochemistry and clinical prognostic factors in tamoxifen-treated estrogen receptor-positive breast cancer. Clin Cancer Res 2010;16:5222-32.

6. Jerevall PL, Ma XJ, Li H, Salunga R, Kesty NC, Erlander MG, et al. Prognostic utility of HOXB13:IL17BR and molecular grade index in early-stage breast cancer patients from the Stockholm trial. Br J Cancer 2011;104:1762-9.

7. Filipits M, Rudas M, Jakesz R, Dubsky P, Fitzal F, Singer CF, et al. A new molecular predictor of distant recurrence in ER-positive, HER2-negative breast cancer adds independent information to conventional clinical risk factors. Clin Cancer Res 2011;17:6012-20.

8. van 't Veer LJ, Dai H, van de Vijver MJ, He YD, Hart AA, Mao M, et al. Gene expression profiling predicts clinical outcome of breast cancer. Nature 2002;415: 530-6.

9. Moran B, Rahman A, Palonen K, Lanigan FT, Gallagher WM. Master transcriptional regulators in cancer: discovery via reverse engineering approaches and subsequent validation. Cancer Res 2017;77:2186-90.

10. Sotiriou C, Wirapati P, Loi S, Harris A, Fox S, Smeds J, et al. Gene expression profiling in breast cancer: understanding the molecular basis of histologic grade to improve prognosis. J Natl Cancer Inst 2006;98:262-72.

11. Lanigan F, Brien GL, Fan Y, Madden SF, Jerman E, Maratha A, et al. Delineating transcriptional networks of prognostic gene signatures refines treatment recom-
Development of methodology: R. Buus, I. Sestak, S. Barron, C.-J.A. Wang, D. O'Leary, W.M. Gallagher, J. Cuzick

Acquisition of data (provided animals, acquired and managed patients, provided facilities, etc.): R. Buus, T. Loughman, B. Fender, C.L. Ruiz, P. Dynoodt, C.-J.A. Wang, D. O'Leary, W.M. Gallagher, M. Dowsett, J. Cuzick

Analysis and interpretation of data (e.g., statistical analysis, biostatistics, computational analysis): R. Buus, I. Sestak, S. Barron, T. Loughman, D. O'Leary, W.M. Gallagher, M. Dowsett, J. Cuzick

Writing, review, and/or revision of the manuscript: R. Buus, I. Sestak, S. Barron, C.J.A. Wang, D. O'Leary, W.M. Gallagher, M. Dowsett, J. Cuzick

Administrative, technical, or material support (i.e., reporting or organizing data, constructing databases): R. Buus, I. Sestak, C.-J.A. Wang, W.M. Gallagher Study supervision: C.-J.A. Wang, D. O'Leary, W.M. Gallagher

\section{Acknowledgments}

This work was supported by Breast Cancer Now, working in partnership with Walk the Walk and by the Royal Marsden National Institutes of Health Biomedical Research Centre and Cancer Research UK grant awarded to J. Cuzick (C569/A16891). W.M. Gallagher was supported by the Irish Cancer Society Collaborative Cancer Research Centre BREAST-PREDICT (CCRC13GAL) and Science Foundation Ireland under grant no. 15/IA/3104. OncoMark received funding from the European Union's Horizon 2020 research and innovation programme under grant agreement no. 698630

The costs of publication of this article were defrayed in part by the payment of page charges. This article must therefore be hereby marked advertisement in accordance with 18 U.S.C. Section 1734 solely to indicate this fact.

Received March 1, 2019; revised July 1, 2019; accepted October 18, 2019; published first October 22, 2019.

mendations for lymph node-negative breast cancer patients. FEBS J 2015;282: 3455-73.

12. Bergamaschi A, Madak-Erdogan Z, Kim YJ, Choi YL, Lu H, Katzenellenbogen BS. The forkhead transcription factor FOXM1 promotes endocrine resistance and invasiveness in estrogen receptor-positive breast cancer by expansion of stem-like cancer cells. Breast Cancer Res 2014;16:436.

13. Liao YC, Ruan JW, Lua I, Li MH, Chen WL, Wang JR, et al. Overexpressed hPTTG1 promotes breast cancer cell invasion and metastasis by regulating GEFH1/RhoA signalling. Oncogene 2012;31:3086-97.

14. Talvinen K, Karra H, Hurme S, Nykanen M, Nieminen A, Anttinen J, et al. Securin promotes the identification of favourable outcome in invasive breast cancer. Br J Cancer 2009;101:1005-10.

15. Jain M, Zhang L, Boufraqech M, Liu-Chittenden Y, Bussey K, Demeure MJ, et al. ZNF367 inhibits cancer progression and is targeted by miR-195. PLoS One 2014; 9:e101423.

16. Cuzick J, Sestak I, Baum M, Buzdar A, Howell A, Dowsett M, et al. Effect of anastrozole and tamoxifen as adjuvant treatment for early-stage breast cancer: 10-year analysis of the ATAC trial. Lancet Oncol 2010;11: 1135-41.

17. Dowsett M, Cuzick J, Wale C, Forbes J, Mallon EA, Salter J, et al. Prediction of risk of distant recurrence using the 21 -gene recurrence score in nodenegative and node-positive postmenopausal patients with breast cancer treated with anastrozole or tamoxifen: a TransATAC study. J Clin Oncol 2010;28:1829-34

18. Dowsett M, Sestak I, Lopez-Knowles E, Sidhu K, Dunbier AK, Cowens JW, et al. Comparison of PAM50 risk of recurrence score with oncotype DX and IHC4 for predicting risk of distant recurrence after endocrine therapy. J Clin Oncol 2013; 31:2783-90.

19. Sgroi DC, Sestak I, Cuzick J, Zhang Y, Schnabel CA, Schroeder B, et al. Prediction of late distant recurrence in patients with oestrogen-receptor-positive breast cancer: a prospective comparison of the breast-cancer index (BCI) assay, 21-gene recurrence score, and IHC4 in the TransATAC study population. Lancet Oncol 2013;14:1067-76.

20. Buus R, Sestak I, Kronenwett R, Denkert C, Dubsky P, Krappmann K, et al. Comparison of EndoPredict and EPclin with oncotype DX recurrence score for 
OncoMasTR is Prognostic in ER ${ }^{+} / \mathrm{HER2}^{-}$Breast Cancer

prediction of risk of distant recurrence after endocrine therapy. J Natl Cancer Inst 2016;108:djw149.

21. Cuzick J, Dowsett M, Pineda S, Wale C, Salter J, Quinn E, et al. Prognostic value of a combined estrogen receptor, progesterone receptor, Ki-67, and human epidermal growth factor receptor 2 immunohistochemical score and comparison with the genomic health recurrence score in early breast cancer. J Clin Oncol 2011;29:4273-8.
22. Sparano JA, Gray RJ, Makower DF, Pritchard KI, Albain KS, Hayes DF, et al. Adjuvant chemotherapy guided by a 21-gene expression assay in breast cancer. N Engl J Med 2018;379:111-21.

23. Dowsett M, Sestak I, Buus R, Lopez-Knowles E, Mallon E, Howell A, et al. Estrogen receptor expression in 21-gene recurrence score predicts increased late recurrence for estrogen-positive/HER2-negative breast cancer. Clin Cancer Res 2015;21:2763-70. 
AACR American Association for Cancer Research

\section{Clinical Cancer Research}

\section{Validation of the OncoMasTR Risk Score in Estrogen Receptor-Positive/HER2-Negative Patients: A TransATAC study}

Richard Buus, Ivana Sestak, Stephen Barron, et al.

Clin Cancer Res Published OnlineFirst October 22, 2019. $\begin{array}{ll}\text { Updated version } & \text { Access the most recent version of this article at: } \\ \text { doi:10.1158/1078-0432.CCR-19-0712 }\end{array}$

Supplementary Access the most recent supplemental material at:

Material http://clincancerres.aacrjournals.org/content/suppl/2019/10/22/1078-0432.CCR-19-0712.DC1

E-mail alerts Sign up to receive free email-alerts related to this article or journal.

Reprints and To order reprints of this article or to subscribe to the journal, contact the AACR Publications

Subscriptions Department at pubs@aacr.org.

Permissions To request permission to re-use all or part of this article, use this link

http://clincancerres.aacrjournals.org/content/early/2019/12/13/1078-0432.CCR-19-0712.

Click on "Request Permissions" which will take you to the Copyright Clearance Center's

(CCC)

Rightslink site. 